\title{
Dynamical morphology of the brain's ventricular cavities in hydrocephalus
}

\author{
C. S. DRAPACA*†, S. SIVALOGANATHAN $\dagger$, G. TENTI $\dagger$ and J. M. DRAKE $\ddagger$ \\ $\dagger$ Department of Applied Mathematics, University of Waterloo, Waterloo, ON, Canada, N2L 3G1 \\ $\ddagger$ Division of Neurosurgery, The Hospital for Sick Children, University of Toronto, Toronto, ON, Canada, M5G 1X8 \\ (Received 4 February 2003; revised 30 September 2004; in final form 31 March 2005)
}

\begin{abstract}
Although interest in the biomechanics of the brain goes back over centuries, mathematical models of hydrocephalus and other brain abnormalities are still in their infancy and a much more recent phenomenon. This is rather surprising, since hydrocephalus is still an endemic condition in the pediatric population with an incidence of approximately $1-3$ per 1000 births. Treatment has dramatically improved over the last three decades, thanks to the introduction of cerebrospinal fluid (CSF) shunts. Their use, however, is not without problems and the shunt failure at two years remains unacceptably high at $50 \%$. The most common factor causing shunt failure is obstruction, especially of the proximal catheters. There is currently no agreement among neurosurgeons as to the optimal catheter tip position; however, common sense suggests that the lowest risk location is the place that remains larger after ventricular decompression drainage. Thus, success in this direction will depend on the development of a quantitative theory capable of predicting the ultimate shape of the ventricular wall. In this paper, we report on some recent progress towards the solution to this problem.
\end{abstract}

Keywords: Hydrocephalus; Brain biomechanics; Ventricular shunt location; Mathematical models

\section{Introduction}

Hydrocephalus is a clinical condition, which occurs when normal cerebrospinal fluid (CSF) circulation is impeded within the cranial cavity. Normally, there is a delicate balance between the rate of formation and of absorption of the CSF, the entire volume being absorbed and replaced once every $12-24 \mathrm{~h}$ [1].

On the basis of extensive data concerning the migration of dyes, radioisotopes and other tracers injected into the CSF, it is now established that the CSF which is formed by active secretion by the choroid plexus, circulates along the craniospinal pathways to be absorbed predominantly via arachnoid granulations into the superior sagittal sinus [2-4]. The rate of absorption is proportional to the pressure gradient between pressure in the subarachnoid CSF space and venous pressure in the sagittal sinus. This pressure may be enhanced by the arterial pulsations of the brain with a small movement towards the superior sagittal sinus with each heartbeat. Hence, the CSF flow is neither slow nor steady [5]. An increase in CSF-production, an obstruction of CSFcirculation, or an obstruction of the venous outflow system may cause hydrocephalus [6]. As a result, in a hydrocephalic brain, the amount of CSF increases while the amount of white matter is compressed, producing a symmetrical dilatation of the ventricular system as can be seen in figure 1a [7].

The incidence of hydrocephalus, regardless of type and irrespective of race, sex or geographic differences, is approximately 1-3 per 1000 births. Untreated hydrocephalus has a poor natural history, with a death rate of 20 to $25 \%$ and severe physical and mental disabilities in survivors [8]. The efforts in treatments have been principally through CSF fluid diversion. More recently, different types of extra-cranial shunting and other innovative mechanical techniques have been extensively used. The shunt mechanics is based mainly on the difference in pressure between the inlet (ventricular) pressure and outlet (peritoneal) pressure; the shunt will start to drain the excess CSF when the ventricular pressure acting on it exceeds some threshold [9]. Within limits (thanks to the elastic properties of the brain parenchyma), the dilatation of the ventricles can be reversed by shunting procedures as can be seen in figure $1 \mathrm{~b}$ showing the same hydrocephalic brain as in figure 1a 3 months after shunting. Unfortunately, even though CSF shunting appears to be an effective treatment, the rate of shunt

*Corresponding author. Email: drapaca.corina@mayo.edu

Journal of Theoretical Medicine

ISSN 1027-3662 print/ISSN 1607-8578 online (C) 2005 Taylor \& Francis

http://www.tandf.co.uk/journals

DOI: $10.1080 / 10273660500143631$ 


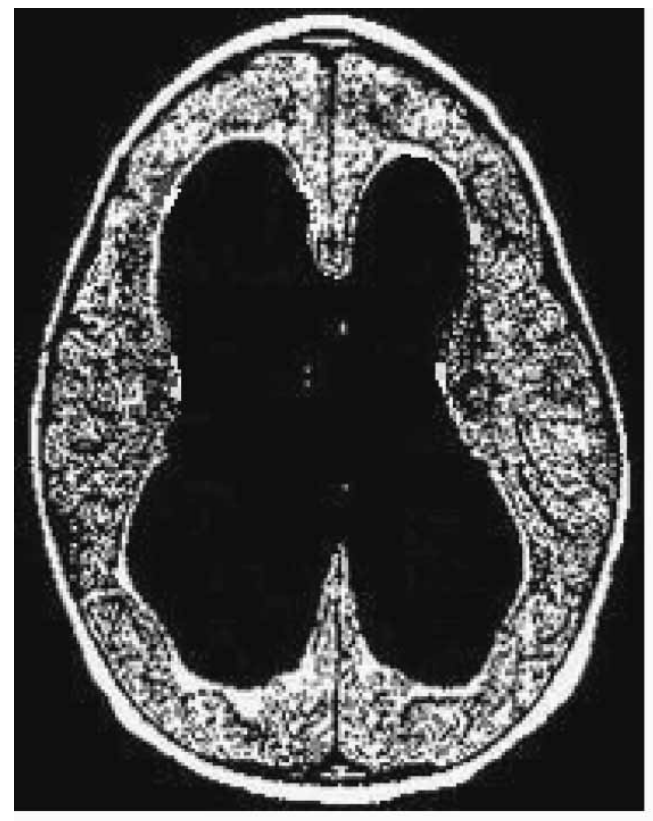

a

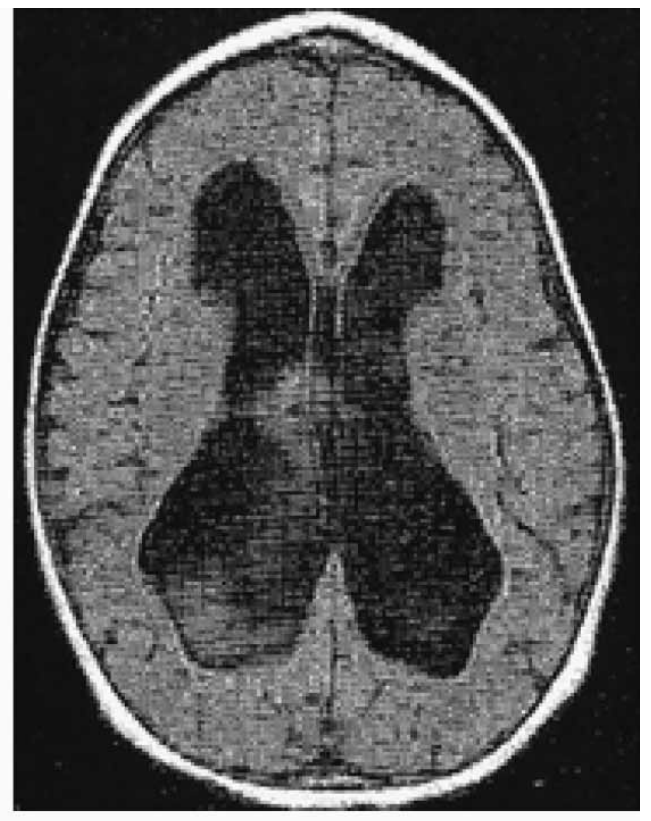

b

Figure 1. (a) Horizontal section of a hydrocephalic brain before shunt implantation. (b) Horizontal section of a hydrocephalic brain 3 months after shunt implantation.

failure is still unacceptably high with less than $50 \%$ of the shunts still functioning after 10 years [6].

The three main problems of shunting are (1) shunt obstruction, (2) infection and (3) over/under drainage of CSF [10]. The most common factor causing shunt failures is obstruction, especially of the proximal catheters $[11,12]$. There is currently no agreement among neurosurgeons as to the optimal catheter tip position; however, common sense suggests that the lowest risk location is the place that remains larger after ventricular decompression drainage $[9,10,13,14]$. Thus, success in this direction will depend on the development of a quantitative theory capable of predicting the ultimate shape of the ventricular wall.

In this paper, we report on some recent progress towards the solution of such a problem. To simplify the mathematics, we consider the two dimensional case depicted in figure 1a, which reproduces a typical horizontal CT scan of a 3 year old hydrocephalic child. The intersection of the ventricular wall with the horizontal plane is the ventricular CSF-tissue boundary, which is a non-convex simple closed curve. By using image processing techniques based on chain encoding and elliptical Fourier descriptors, we construct a parametric representation of this curve in section 2 . In the next three sections we present three different methods-namely the Lagrangian (section 3), perturbation (section 4) and level set (section 5) methods-which may be used to describe the time evolution of the ventricular wall position when the wall speed is given. In section 6, we apply these methods to hydrocephalus (more precisely, to the curve representing the ventricular CSF-tissue boundary) and conclude that the level set method is the most reliable method. Finally, we discuss the results and the prospects for future improvements in the last section.

As we have mentioned before, all the methods used in our numerical simulation of hydrocephalus require that we know beforehand the velocity of each point of the ventricular wall as it moves inwardly under the decreasing pressure gradient produced by the shunting process. Finding the ventricular decompression speed is not an easy task and is still an open problem because it may depend on factors like the initial ventricular size, reconfiguration of the cerebral mantle with ventricular decompression by the shunt, cranium growth in young children, the intrinsic stiffness of the catheter [12]. Taking into account, the lack of information concerning this ventricular decompression speed we will consider for simplicity the two easiest possible cases: (1) when the wall speed is constant and (2) when the speed is linearly dependent on the curvature at each point of the ventricular wall. Case (2) was inspired by the fact that, intuitively, the evolution of any point on the ventricular wall will be affected by the evolution of its neighbours, and hence by the local bending (curvature) of the ventricular wall. Thus, a speed which is linearly dependent on the curvature of the ventricular wall is an easier case worthwhile investigating.

\section{Chain encoding and elliptical Fourier descriptors}

One of the modern processes which allows us to achieve an accurate outline of a given image is called digitization [15]. Digitization may be viewed schematically as a method for placing a grid of sufficiently fine mesh over the outline of an image and then defining an $m \times n$ incidence matrix $M$, 
of 0 's and 1's, to represent the image, where a 1 in the $(i, j)$ position of $M$ denotes the fact that the outline intersects the $(i, j)$ grid cell of the mesh. For example, for the contour given in figure $2 \mathrm{a}$ one possible digitized image associated to it is the matrix shown in figure $2 \mathrm{c}$.

The digitized data may then be given a representation through a so-called chain code which identifies the direction one goes from the current cell with a 1 to an adjacent cell with a 1, and so on. Any continuous closed contour can be approximated by a chain code which is a sequence of piecewise linear fits that consist of eight standardized line segments [16]. The code of a contour is then the chain $V$ of length $K, V=a_{1} a_{2} \ldots a_{K}$, where each link $a_{i}$ is an integer between 0 and 7 and corresponds to a directed line segment of length 1 or $\sqrt{2}$ in the $x y$-plane, the directions of the segments being multiples of $45^{\circ}$. Figure 3 gives the numeration scheme for the chain code.

The properties of a chain code are easy to derive. If $V=a_{1} a_{2} \ldots a_{K}$ is a chain code then its total arc length $S$ is given by

$$
S=\sum_{i=1}^{K}\left[1+\frac{\sqrt{2}-1}{2}\left(1-(-1)^{a_{i}}\right)\right] .
$$

Let $s(0 \leq s \leq S)$ be the arc length of the unique piecewise linear line-type image $v$ associated with the chain code $V$. Then $v=v(s)=(x(s), y(s))$, where $x(s)$ and $y(s)$ specify the $x$ and $y$ coordinates, respectively, of $v ; x(s)$ and $y(s)$ are called the $x$ and y projections, respectively. The time $\Delta s_{i}$ required to traverse a particular link $a_{i}$ in the assumption that the chain code is followed at constant speed, is equal to 1 if $a_{i}$ is an even number or is equal to $\sqrt{2}$ if $a_{i}$ is odd. The time required to traverse the first $p$ links in the chain is

$$
s_{p}=\sum_{i=1}^{p} \Delta s_{i}
$$

The changes in the $x$ and $y$ projections of the image induced by the chain as the link $a_{i}$ is traversed are $\Delta x_{i}=$ $\operatorname{sgn}\left(6-a_{i}\right) \operatorname{sgn}\left(2-a_{i}\right), \Delta y_{i}=\operatorname{sgn}\left(4-a_{i}\right) \operatorname{sgn}\left(a_{i}\right)$, where

$$
\operatorname{sgn}(z)=\left\{\begin{array}{cl}
1 & \text { if } z>0 \\
0 & \text { if } z=0 \\
-1 & \text { if } z<0
\end{array}\right.
$$
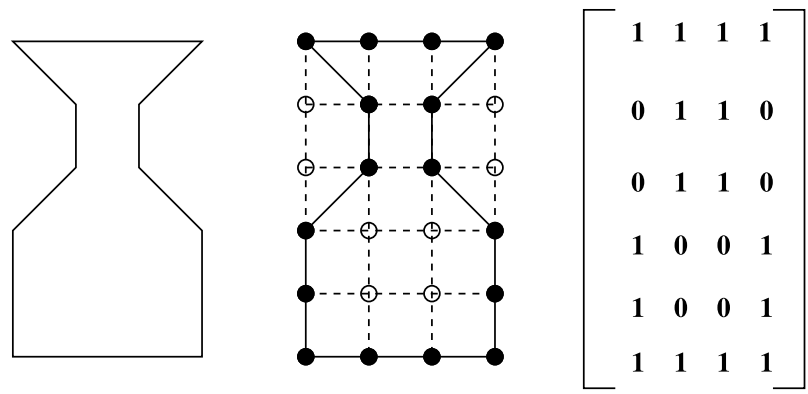

b

\section{c}

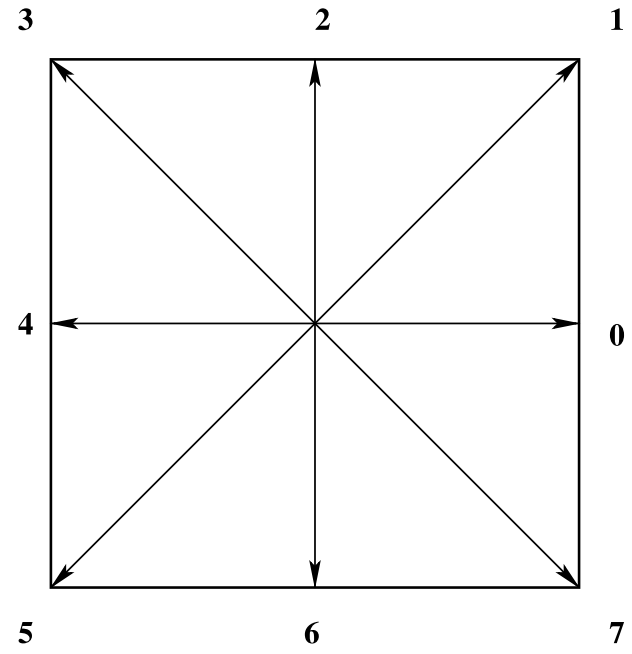

Figure 3. Chain-code line segments.

For example, if the direction chosen to pass from one cell with a 1 to an adjacent cell with a 1 is clockwise, then the chain code corresponding to figure $2 \mathrm{a}$ is $V=0005676644422123$ and it is shown in figure 4.

The Fourier series representation of a chain-encoded contour has the following parametric form:

$$
x(s)=A_{0}+\sum_{n=1}^{N}\left[A_{n} \cos \frac{2 n \pi s}{S}+B_{n} \sin \frac{2 n \pi s}{S}\right],
$$

$$
y(s)=C_{0}+\sum_{n=1}^{N}\left[C_{n} \cos \frac{2 n \pi s}{S}+D_{n} \sin \frac{2 n \pi s}{S}\right],
$$

where $n$ equals the harmonic number, $N$ equals the maximum harmonic number and the coefficients $A_{0}, A_{n}$,

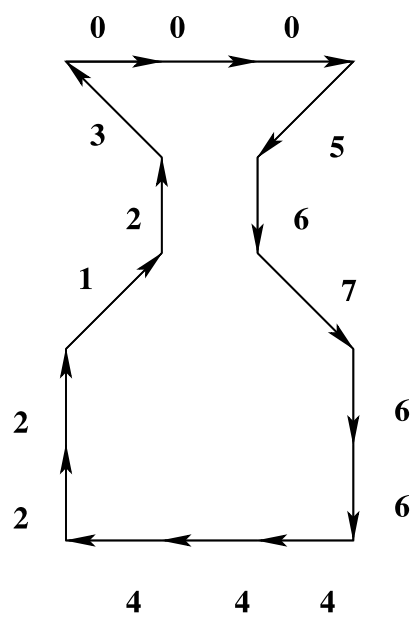

Figure 4. Chain code of the image.

Figure 2. Example of an image (a), one of its associated grids (b) and one of its digitization matrices (the 1's correspond to the black circles of the grid while the 0 's correspond to the white circles) (c). 
$B_{n}, C_{0}, C_{n}, D_{n}$ are given by:

$$
\begin{aligned}
& A_{n}= \frac{S}{2 n^{2} \pi^{2}} \sum_{p=1}^{K} \frac{\Delta x_{p}}{\Delta s_{p}}\left[\cos \frac{2 n \pi s_{p}}{S}-\cos \frac{2 n \pi s_{p-1}}{S}\right], \\
& B_{n}= \frac{S}{2 n^{2} \pi^{2}} \sum_{p=1}^{K} \frac{\Delta x_{p}}{\Delta s_{p}}\left[\sin \frac{2 n \pi s_{p}}{S}-\sin \frac{2 n \pi s_{p-1}}{S}\right], \\
& C_{n}= \frac{S}{2 n^{2} \pi^{2}} \sum_{p=1}^{K} \frac{\Delta y_{p}}{\Delta s_{p}}\left[\cos \frac{2 n \pi s_{p}}{S}-\cos \frac{2 n \pi s_{p-1}}{S}\right], \\
& D_{n}= \frac{S}{2 n^{2} \pi^{2}} \sum_{p=1}^{K} \frac{\Delta y_{p}}{\Delta s_{p}}\left[\sin \frac{2 n \pi s_{p}}{S}-\sin \frac{2 n \pi s_{p-1}}{S}\right], \\
& C_{0}=\frac{1}{S} \sum_{p=1}^{K}\left[\frac{\Delta y_{p}}{2 \Delta s_{p}}\left(s_{p}^{2}-s_{p-1}^{2}\right)+\delta_{p}\left(s_{p}-s_{p-1}\right),\right. \\
& A_{0}=\frac{1}{S} \sum_{p=1}^{K}\left[\frac{\Delta x_{p}}{2 \Delta s_{p}}\left(s_{p}^{2}-s_{p-1}^{2}\right)+\xi_{p}\left(s_{p}-s_{p-1}\right)\right],
\end{aligned}
$$

with

$$
\begin{aligned}
\xi_{1} & =\delta_{1}=0, \\
\xi_{p} & =\sum_{j=1}^{p-1} \Delta x_{j}-\frac{\Delta x_{p}}{\Delta s_{p}} \sum_{j=1}^{p-1} \Delta s_{j}, \\
\delta_{p} & =\sum_{j=1}^{p-1} \Delta y_{j}-\frac{\Delta y_{p}}{\Delta s_{p}} \sum_{j=1}^{p-1} \Delta s_{j} .
\end{aligned}
$$

The convergence of the Fourier series (2.1) and (2.2) is, in general, fast and can be achieved with fewer harmonics than $N[16,17]$. For example, for the chain code $V=$ 0005676644422123 the Fourier series (2.1) and (2.2) converge to the given image in only four harmonics (see figure 5).

The chain codes are dependent upon size, orientation, initial point and the grid mesh used in the digitization of
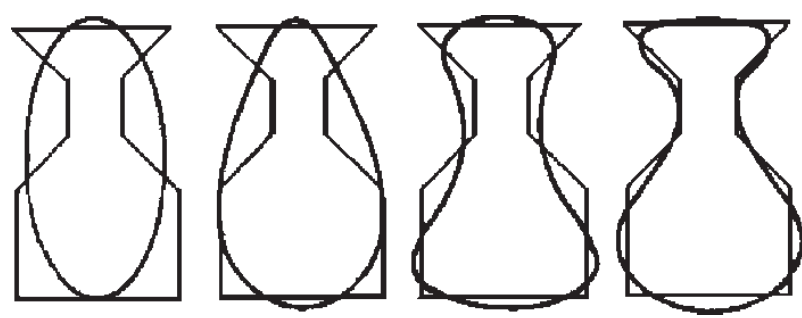

Figure 5. First four harmonic approximations of the chain code $V$. the given image. The components

$$
x_{n}(s)=A_{n} \cos \frac{2 n \pi s}{S}+B_{n} \sin \frac{2 n \pi s}{S},
$$

and

$$
y_{n}(s)=C_{n} \cos \frac{2 n \pi s}{S}+D_{n} \sin \frac{2 n \pi s}{S}
$$

describe elliptical contours. For this reason the Fourier representations (2.1) and (2.2) are called elliptical Fourier series. The ellipse defined by the first pair of components $x_{1}(s)$ and $y_{1}(s)$ can be used to make the representation free of size and orientation and we implemented this in our numerical algorithm. The representation becomes independent of translation or initial point for tracing around an outline by ignoring the terms $A_{0}$ and $C_{0}$.

\section{Lagrangian method}

We consider a simple, smooth, closed initial curve (or front) $v(0)$ in $\mathbf{R}^{2}$ whose parametric representation is given by the elliptical Fourier series. Let $v(t)$ be the oneparameter family of curves, where $t \in[0, \infty)$ is time, generated by moving the initial curve along its normal vector field with speed $F$, a given function of curvature [18]. We denote by $\vec{x}(s, t)$ the position vector which parameterizes $v(t)$ by $s, 0 \leq s \leq S, \vec{x}(0, t)=\vec{x}(S, t)$. Following [18], we parameterize the curve so that $s$ increases in the counter-clockwise direction. If $\kappa(s, t)$ is the curvature at $\vec{x}(s, t)$, classical kinematics gives

$$
\begin{aligned}
& \vec{n}(s, t) \cdot \frac{\partial \vec{x}(s, t)}{\partial t}=F(\kappa(s, t)), \\
& \vec{x}(s, 0)=v(0) \text { prescribed; } \\
& s \in[0, S], t \in[0, \infty)
\end{aligned}
$$

where $\vec{n}(s, t)$ is the external unit normal vector at $\vec{x}(s, t)$. Written in terms of the coordinates $\vec{x}(s, t)=$ $(x(s, t), y(s, t))$, an equivalent formulation is

$$
\begin{gathered}
x_{t}=F\left(\frac{y_{s s} x_{s}-x_{s s} y_{s}}{\left(x_{s}^{2}+y_{s}^{2}\right)^{3 / 2}}\right) \frac{y_{s}}{\sqrt{\left(x_{s}^{2}+y_{s}^{2}\right)}} \\
y_{t}=F\left(\frac{y_{s s} x_{s}-x_{s s} y_{s}}{\left(x_{s}^{2}+y_{s}^{2}\right)^{3 / 2}}\right) \frac{-x_{s}}{\sqrt{\left(x_{s}^{2}+y_{s}^{2}\right)}} \\
(x(s, 0), y(s, 0))=v(0), 0 \leq s \leq S
\end{gathered}
$$

where $\kappa(s, t)=\left(y_{s s} x_{s}-x_{s s} y_{s}\right) /\left(x_{s}^{2}+y_{s}^{2}\right)^{3 / 2}$ and where we denoted by $f_{s}=\partial f / \partial s, f_{t}=\partial f / \partial t$.

This is a Lagrangian representation because the range of $(x(s, t), y(s, t))$ describes the moving front.

In the case when the front moves at a constant speed $F=-1$ (in arbitrary units) the solution of the 
system (3.2) is

$$
\begin{gathered}
x(s, t)=-\frac{y_{s}(s, t=0)}{\sqrt{\left(x_{s}^{2}(s, t=0)+y_{s}^{2}(s, t=0)\right)}} t+x(s, t=0) \\
y(s, t)=\frac{x_{s}(s, t=0)}{\sqrt{\left(x_{s}^{2}(s, t=0)+y_{s}^{2}(s, t=0)\right)}} t+y(s, t=0)
\end{gathered}
$$

A front propagating at constant speed can form corners as it evolves. At such points, the front is no longer differentiable and a weak solution must be constructed to continue the solution.

When the front speed is curvature-dependent then only a numerical solution can be found for system (3.2).

A standard approach to modelling moving fronts comes from discretizing the Lagrangian form of the equations of motion given in equation (3.2). In this approach [18], the parameterization is discretized into a set of marker particles whose positions at any time are used to reconstruct the front.

In the present work, the discretization has to be in agreement with the chain code associated with the given front. The parameterization interval $[0, S]$ is divided into $K$ intervals of sizes $\Delta s_{i}, i=1, \bar{K}$, where $\Delta s_{i}$ were defined in the previous section. We obtain in this way $K+1$ mesh points $s_{p}=\sum_{i=1}^{p} \Delta s_{i}, p=1, \bar{K}$ and $s_{K+1}=s_{1}$ (in order to close the curve, the first and the last mesh points must be the same). We divide the time interval into equal intervals of equal length $\Delta t$. The image of each mesh point $s_{i}$ at each time step $n \Delta t$ is a marker point $\left(x_{i}^{n}, y_{i}^{n}\right)$ on the moving front. The goal is to obtain a numerical algorithm that will produce new values $\left(x_{i}^{n+1}, y_{i}^{n+1}\right)$ from the previous positions.

In order to approximate parameter derivatives at each marker point we use central difference schemes based on Taylor series and get

$$
\frac{\mathrm{d} x_{i}^{n}}{\mathrm{~d} s} \approx \frac{x_{i+1}^{n}-x_{i-1}^{n}}{s_{i+1}-s_{i-1}}, \frac{\mathrm{d} y_{i}^{n}}{\mathrm{~d} s} \approx \frac{y_{i+1}^{n}-y_{i-1}^{n}}{s_{i+1}-s_{i-1}}
$$

$$
\frac{\mathrm{d}^{2} x_{i}^{n}}{\mathrm{~d} s^{2}} \approx 2 \frac{\left(s_{i}-s_{i-1}\right) x_{i+1}^{n}-\left(s_{i+1}-s_{i-1}\right) x_{i}^{n}+\left(s_{i+1}-s_{i}\right) x_{i-1}^{n}}{\left(s_{i}-s_{i-1}\right)\left(s_{i+1}-s_{i-1}\right)\left(s_{i+1}-s_{i}\right)}
$$

$$
\frac{\mathrm{d}^{2} y_{i}^{n}}{\mathrm{~d} s^{2}} \approx 2 \frac{\left(s_{i}-s_{i-1}\right) y_{i+1}^{n}-\left(s_{i+1}-s_{i-1}\right) y_{i}^{n}+\left(s_{i+1}-s_{i}\right) y_{i-1}^{n}}{\left(s_{i}-s_{i-1}\right)\left(s_{i+1}-s_{i-1}\right)\left(s_{i+1}-s_{i}\right)}
$$

Time derivatives may be replaced by the forward difference approximations

$$
\frac{\mathrm{d} x_{i}^{n}}{\mathrm{~d} t} \approx \frac{x_{i}^{n+1}-x_{i}^{n}}{\Delta t}, \quad \frac{\mathrm{d} y_{i}^{n}}{\mathrm{~d} t} \approx \frac{y_{i}^{n+1}-y_{i}^{n}}{\Delta t}
$$

Substitution of these approximations into the equation
(3.2) produces the scheme:

$$
\begin{aligned}
\left(x_{i}^{n+1}, y_{i}^{n+1}\right)= & \left(x_{i}^{n}, y_{i}^{n}\right)+\Delta t F\left(\kappa_{i}^{n}\right) \\
& \times \frac{\left(y_{i+1}^{n}-y_{i-1}^{n}\right)-\left(x_{i+1}^{n}-x_{i-1}^{n}\right)}{\left(\left(x_{i+1}^{n}-x_{i-1}^{n}\right)^{2}+\left(y_{i+1}^{n}-y_{i-1}^{n}\right)^{2}\right)^{1 / 2}}
\end{aligned}
$$

where

$$
\begin{aligned}
\kappa_{i}^{n}= & \frac{\left(s_{i+1}-s_{i-1}\right)^{2}}{\left(s_{i+1}-s_{i}\right)\left(s_{i}-s_{i-1}\right)} \\
& \times \frac{1}{\left(\left(x_{i+1}^{n}-x_{i-1}^{n}\right)^{2}+\left(y_{i+1}^{n}-y_{i-1}^{n}\right)^{2}\right)^{3 / 2}} \\
& \times\left[\left(y_{i+1}^{n}-2 y_{i}^{n}+y_{i-1}^{n}\right)\left(x_{i+1}^{n}-x_{i-1}^{n}\right)-\left(x_{i+1}^{n}-2 x_{i}^{n}+x_{i-1}^{n}\right)\right. \\
& \left.\times\left(y_{i+1}^{n}-y_{i-1}^{n}\right)\right]
\end{aligned}
$$

is the curvature at point $\left(x_{i}^{n}, y_{i}^{n}\right)$.

Using the fact that the curve is closed, the above numerical scheme (3.4) updates all the positions of the particles from one time step to the next.

\section{Perturbation method}

We consider again equation (3.2) with a speed function linearly dependent on the curvature of the given front: $F=-1-\epsilon \kappa$.

As we will see later in the application section, the Lagrangian method for this kind of speed function is unstable even for sufficiently small $\epsilon$.

In this section we try to improve the Lagrangian method for $\epsilon \ll 1$ by using a perturbation method technique.

Thus we look for a solution to equation (3.2) of the form [19]:

$$
\begin{aligned}
& x(s, t)=x_{0}(s, t)+\epsilon x_{1}(s, t)+\mathcal{O}\left(\epsilon^{2}\right), \\
& y(s, t)=y_{0}(s, t)+\epsilon y_{1}(s, t)+\mathcal{O}\left(\epsilon^{2}\right)
\end{aligned}
$$

If we substitute equation (4.1) into the expression of the curvature $\kappa(s, t)=\left(y_{s s} x_{s}-x_{s s} y_{s}\right) /\left(x_{s}^{2}+y_{s}^{2}\right)^{3 / 2}$ and we assume that

$$
2 \epsilon \frac{x_{0_{s}} x_{1_{s}}+y_{0_{s}} y_{1_{s}}}{x_{0_{s}}^{2}+y_{0_{s}}^{2}}<1
$$

then we get the following expression for $\kappa$ :

$$
\kappa \approx \alpha_{0}(s, t)+\epsilon \alpha_{1}(s, t)
$$


where

$$
\begin{aligned}
\alpha_{0}(s, t)=\frac{y_{0_{s s}} x_{0_{s}}-x_{0_{s s}} y_{0_{s}}}{\left(x_{0_{s}}^{2}+y_{0_{s}}^{2}\right)^{3 / 2}} \\
\alpha_{1}(s, t)=-\frac{1}{\left[x_{0_{s}}^{2}+y_{0_{s}}^{2}\right]^{3 / 2}} \\
\times\left[y_{1_{s s}} x_{0_{s}}+y_{0_{s s}} x_{1_{s}}-x_{1_{s s}} y_{0_{s}}-y_{1_{s}} x_{0_{s s}}\right. \\
\left.-3\left(y_{0_{s s}} x_{0_{s}}-x_{0_{s s}} y_{0_{s}}\right) \frac{x_{0_{s}} x_{1_{s}}+y_{0_{s}} y_{1_{s}}}{x_{0_{s}}^{2}+y_{0_{s}}^{2}}\right]
\end{aligned}
$$

Also, if we use again equation (4.1) and power series properties, we get:

$$
\frac{1}{\left(x_{s}^{2}+y_{s}^{2}\right)^{1 / 2}}=\beta_{0}(s, t)+\epsilon \beta_{1}(s, t)
$$

where

$$
\begin{aligned}
& \beta_{0}(s, t)=\frac{1}{\left(x_{0_{s}}^{2}+y_{0_{s}}^{2}\right)^{1 / 2}} \\
& \beta_{1}(s, t)=-\frac{1}{\left(x_{0_{s}}^{2}+y_{0_{s}}^{2}\right)^{1 / 2}} \frac{x_{0_{s}} x_{1_{s}}+y_{0_{s}} y_{1_{s}}}{x_{0_{s}}^{2}+y_{0_{s}}^{2}}
\end{aligned}
$$

If we now substitute equations (4.1)-(4.3) into equation (3.2) we obtain:

$$
\begin{array}{r}
x_{0_{t}}+\epsilon x_{1_{t}}=-\beta_{0} y_{0_{s}}-\epsilon\left[\beta_{0}\left(y_{1_{s}}+\alpha_{0} y_{0_{s}}\right)+\beta_{1} y_{0_{s}}\right] \\
y_{0_{t}}+\epsilon y_{1_{t}}=\beta_{0} x_{0_{s}}+\epsilon\left[\beta_{0}\left(x_{1_{s}}+\alpha_{0} x_{0_{s}}\right)+\beta_{1} x_{0_{s}}\right]
\end{array}
$$

Thus, from equation (4.4), $\epsilon^{0}$-equations are:

$$
\begin{gathered}
x_{0_{t}}=-\beta_{0} y_{0_{s}}=\frac{-y_{0_{s}}}{\left(x_{0_{s}}^{2}+y_{0_{s}}^{2}\right)^{1 / 2}} \\
y_{0_{t}}=\beta_{0} x_{0_{s}}=\frac{x_{0_{s}}}{\left(x_{0_{s}}^{2}+y_{0_{s}}^{2}\right)^{1 / 2}}
\end{gathered}
$$

which are exactly equation (3.2) for $F=-1$ whose analytical solutions are given by equation (3.3). The $\epsilon^{1}-$ equations are:

$$
\begin{gathered}
x_{1_{t}}=\gamma_{0}\left[x_{0_{s}} y_{1_{s}}-x_{1_{s}} y_{0_{s}}\right]-\alpha_{0} \beta_{0} y_{0_{s}} \\
y_{1_{t}}=\delta_{0}\left[x_{0_{s}} y_{1_{s}}-x_{1_{s}} y_{0_{s}}\right]+\alpha_{0} \beta_{0} x_{0_{s}}
\end{gathered}
$$
where we denoted by $\gamma_{0}=-x_{0_{s}}\left(x_{0_{s}}^{2}+y_{0_{s}}^{2}\right)^{-3 / 2}$,
$\delta_{0}=-y_{0_{s}}\left(x_{0_{s}}^{2}+y_{0_{s}}^{2}\right)^{-3 / 2}$.

The parabolic system (4.6) does not have analytical solutions. To find numerical solutions for equation (4.6) we will use an implicit Cranck-Nicholson scheme which for parabolic systems is, in general, a stable numerical scheme [20].

We discretize the time interval of interest and the parameter interval $[0, S]$ in the same way as in the previous section. Let $x_{j}^{i}=x\left(t_{i}, s_{j}\right), y_{j}^{i}=y\left(t_{i}, s_{j}\right)$, and using a forward difference approximation for the time derivatives and an implicit Crank-Nicholson approximation for the parameter derivatives we obtain the following algorithm to get numerical solutions of system (4.6):

$$
\begin{aligned}
& x_{1_{j}}^{i+1}=x_{1_{j}}^{i}+\frac{\Delta t}{2}\left[\left(\gamma_{0_{j}}^{i+1}\left[x_{0_{s_{j}}}^{i+1} \frac{y_{1_{j+1}}^{i+1}-y_{1_{j}}^{i+1}}{\Delta s_{j+1}}-y_{0_{s_{j}}}^{i+1} \frac{x_{1_{j+1}}^{i+1}-x_{1_{j}}^{i+1}}{\Delta s_{j+1}}\right]\right.\right. \\
& \left.-\left(\alpha_{0} \beta_{0} y_{0_{s}}\right)_{j}^{i+1}\right) \\
& \left.+\left(\gamma_{0_{j}}^{i}\left[x_{0_{s_{j}}^{i}}^{i} \frac{y_{1_{j+1}}^{i}-y_{1_{j}}^{i}}{\Delta s_{j+1}}-y_{0_{s_{j}}}^{i} \frac{x_{1_{j+1}}^{i}-x_{1_{j}}^{i}}{\Delta s_{j+1}}\right]-\left(\alpha_{0} \beta_{0} y_{0_{s}}\right)_{j}^{i}\right)\right] \text {; } \\
& y_{1_{j}}^{i+1}=y_{1 j}^{i}+\frac{\Delta t}{2}\left[\left(\delta_{0_{j}}^{i+1}\left[x_{0_{s_{j}}}^{i+1} \frac{y_{1_{j+1}}^{i+1}-y_{1_{j}}^{i+1}}{\Delta s_{j+1}}-y_{0_{s_{j}}}^{i+1} \frac{x_{1_{j+1}}^{i+1}-x_{1_{j}}^{i+1}}{\Delta s_{j+1}}\right]\right.\right. \\
& \left.+\left(\alpha_{0} \beta_{0} x_{0_{s}}\right)_{j}^{i+1}\right) \\
& \left.+\left(\delta_{0_{j}}^{i}\left[x_{0_{s_{j}}}^{i} \frac{y_{1_{j+1}}^{i}-y_{1_{j}}^{i}}{\Delta s_{j+1}}-y_{0_{s_{j}}}^{i} \frac{x_{1_{j+1}}^{i}-x_{1_{j}}^{i}}{\Delta s_{j+1}}\right]+\left(\alpha_{0} \beta_{0} x_{0_{s}}\right)_{j}^{i}\right)\right]
\end{aligned}
$$

where $x_{0_{s_{j}}}^{i}, y_{0_{s_{i}}}^{i}, \alpha_{0_{j}}^{i}, \beta_{0_{j}}^{i}, \gamma_{0_{j}}^{i}, \delta_{0_{j}}^{i}$ are the values of $x_{0_{s}}, y_{0_{s}}$, $\alpha_{0}, \beta_{0}, \gamma_{0}, \delta_{0}$ for which we have analytical formulas at the points of the constructed mesh.

Finally, the numerical solution of equation (3.2) for the given speed $F=-1-\epsilon \kappa$ with $\epsilon \ll 1$ is given by equations (3.3), (4.1), and (4.7).

\section{Level set method}

First, we will motivate the method presented in this section by a simple example $[18,21]$. We suppose that a circle in the $x y$-plane is an initial front $\Gamma$ at $t=0$ (figure 6a). We imagine that the circle is the level set $\phi=0$ of an initial surface $z=\phi(x, y, t=0)$ in $\mathbf{R}^{3}$ (figure $6 \mathrm{~b}$ ). We can then match the one-parameter family of moving curves $\Gamma(t)$ with a one-parameter family of moving surfaces in such a way that the level set $\phi=0$ always yields the moving front (figures $6 c, d$ ). All that remains is to find an equation of motion for the evolving surface.

In general, let $\Gamma$ be a curve in the plane propagating in a direction normal to itself with speed $F$ such that $\Gamma(t)$ gives the position of the front at time $t$ [18]. We consider that the initial position of the front is the zero level set of a higherdimensional function $\phi$. The evolution of this function $\phi$ and the propagation of the front can be connected through a time dependent initial value problem. At any time the front is given by the zero level set function $\phi(x, y, t)$, i.e. at 


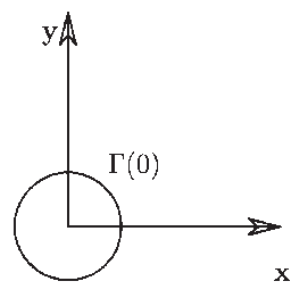

a: Initial Circle

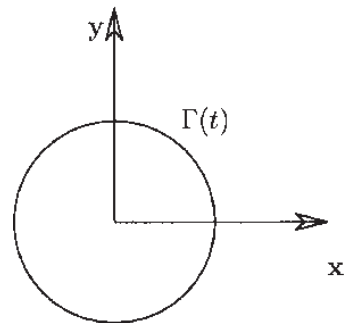

b: Circle at time $\mathbf{t}$
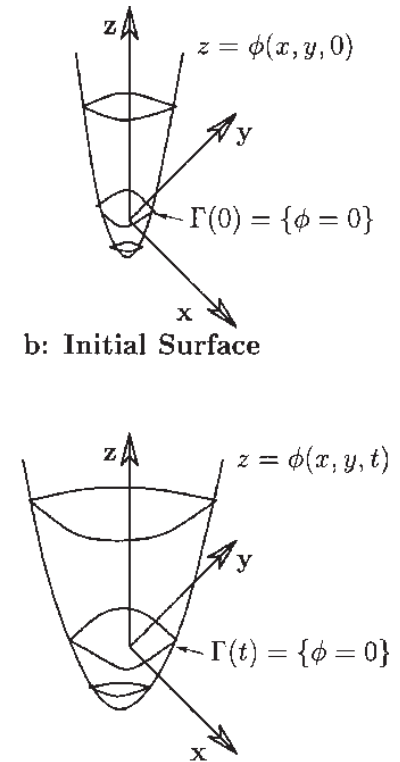

d: Surface at time $t$ b: Initial Surface

Figure 6. Motivational example of the level set method.

any time $t$ the curve is described by the set $\{(x, y) \mid \phi(x, y, t)=0\}$. In order to derive an equation of motion for $\phi$ and match the zero level set of $\phi$ with the evolving curve, we consider a particle moving on the curve with a path given by $(x(t), y(t))$ at time $t$. But at time $t$ the front is given by $\phi(x, y, t)=0$. Since the particle is on the front we must have $\phi(x(t), y(t), t)=0$.

Differentiating with respect to $t$ yields

$$
\phi_{t}+\nabla \phi \cdot\left(x_{t}, y_{t}\right)=0
$$

If we assume that the particle moves with velocity $F \vec{n}$, where $F$ is the curvature dependent speed and $\vec{n}=$ $(\nabla \phi /|\nabla \phi|)$ is the outward unit normal then the velocity is

$$
\left(x_{t}, y_{t}\right)=F \frac{\nabla \phi}{|\nabla \phi|}
$$

From equations (5.1) and (5.2) we get an evolution equation for $\phi$ of the form

$$
\phi_{t}+F|\nabla \phi|=0
$$

where $\phi(x, y, t=0)= \pm d, d$ being the distance from the point $(x, y)$ to the curve at $t=0$. The plus sign is chosen if the point is outside the curve, while the minus sign is chosen if the point is inside the curve.

Equation (5.3) describes the time evolution of the level set function $\phi$ in such a way that the zero level set of this evolving function is identified with the propagating interface.

When $F=-1-\epsilon \kappa$, equation (5.3) becomes:

$$
\phi_{t}-|\nabla \phi|=\epsilon \kappa|\nabla \phi|
$$

Equation (5.4) is a Hamilton-Jacobi equation with viscosity and its numerical solution can be constructed using well-known techniques borrowed from hyperbolic conservation laws [18,19,21,22].

\section{Application to hydrocephalus}

Figure 1a shows an example of a pre-op ventricular wall configuration in a horizontal CT scan of a three year old hydrocephalic brain. As mentioned in the introduction, our first task is to find the parametric expression of this image, using the chain code and the elliptical Fourier series.

We proceed as follows. Using Matlab's image processing toolbox, we display the brain image on the computer screen and extract the ventricular wall which is the closed curve of interest to our numerical simulation (see figure 7). The digitization process, the chain code and the parameterization of the curve are done easily by a Matlab code.

Taking into account the lack of information regarding the speed at which the ventricular wall moves we consider for simplicity the easiest two possible cases:

(i) The speed is constant, $F=-1$.

(ii) The speed at each point depends linearly on the curvature at that point, $F=-1-\epsilon \kappa$ with $\epsilon>0$ a given parameter.

In the end, we compare the images obtained using the Lagrangian method and the level set method in both cases (i) and (ii). Also, in case (ii), for small enough $\epsilon$, we compare results of the Lagrangian method, the

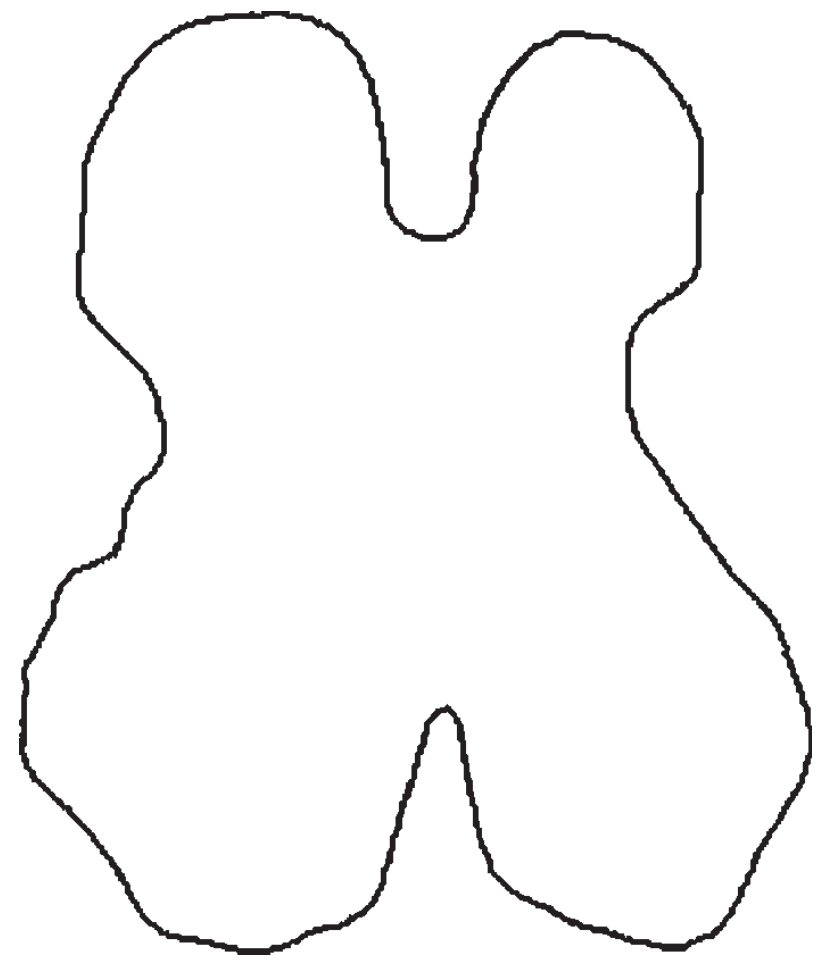

Figure 7. The ventricular CSF-tissue boundary. 

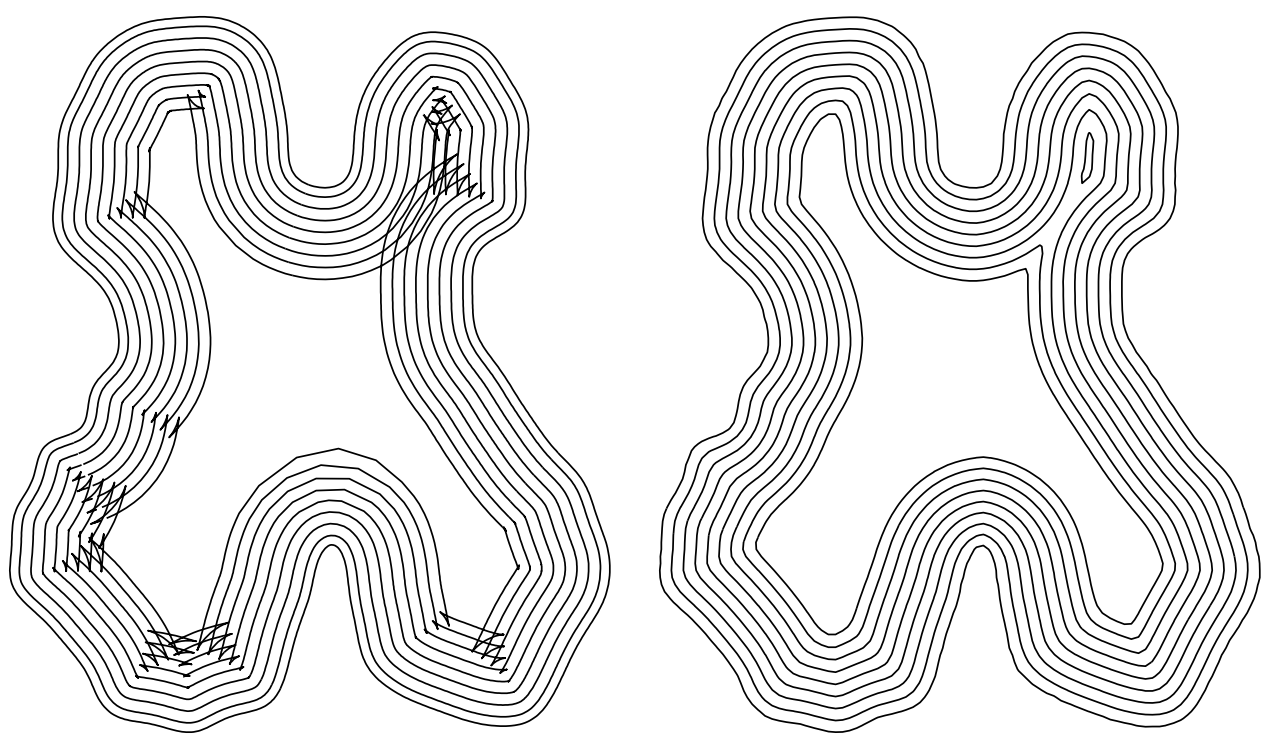

Figure 8. (a) Lagrangian method for $F=-1$. (b) Level set method for $F=-1$.

perturbation method and the level set method. In figures $8-10$ we show the inward evolution of the original curve at eight equally spaced time points.

(i) When $F=-1$, we can easily see that, because the Lagrangian method uses a local (parametric) representation of the front rather than a global one, it is not able to take into account the proper weak solutions when singularities appear. Indeed, if a front which forms a sharp corner moves at constant speed, then we know that an entropy condition must be invoked to produce a reasonable weak solution beyond the formation of the singularity. However, a marker particle approach does not know about the necessary entropy condition, because it uses a local representation of the front in which the swallowtail solution formed by letting the front pass through itself is an acceptable weak solution (see figure 8a).

But from a geometrical point of view it seems that the front at time $t$ should consist of only the set of all points located at distance $t$ from the initial curve [21]. Roughly speaking, we want to remove the tail from the swallowtail.
One way to do this is by taking into account the weak solution which satisfies the following entropy condition:

If the front is viewed as a burning flame, then once a particle is burnt it stays burnt.

This is exactly what the level set method does in order to keep the smoothness of the front at any moment of time (see figure $8 \mathrm{~b}$ ).

(ii) When $F=-1-\epsilon \kappa$, the Lagrangian method becomes unstable even for very small values of $\epsilon$ because of a feedback cycle [18]: (1) small errors in approximate marker positions produce (2) local variations in the computed derivatives leading to (3) variation in the computed particle velocities causing (4) uneven advancement of markers, which yields (5) larger errors in approximate marker positions. Within a few time steps, the small oscillations in the curvature have grown and the computed solution becomes unbounded (see figure 9a).

For small values of $\epsilon$, the perturbation method gives results comparable with those obtained using the level set method. In this particular case, the perturbation method is
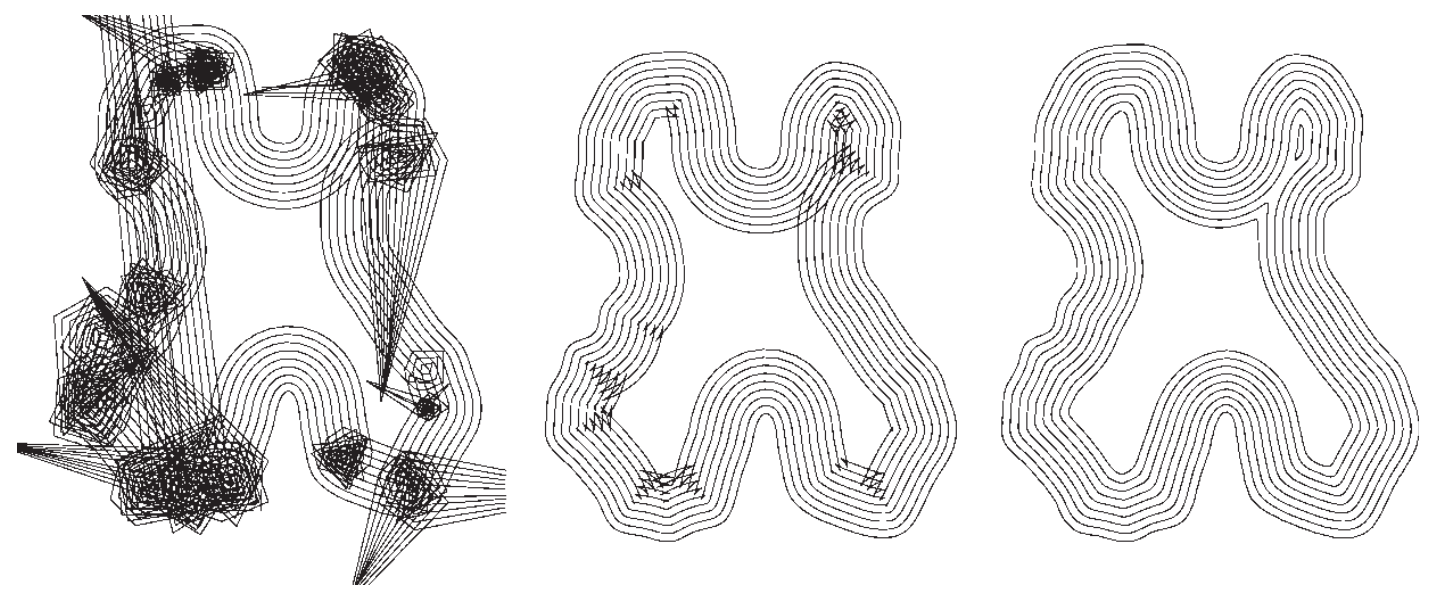

Figure 9. (a) Lagrangian method for $F=-1-0.01 \kappa$. (b) Perturbation method for $F=-1-0.01 \kappa$. (c) Level set method for $F=-1-0.01 \kappa$. 


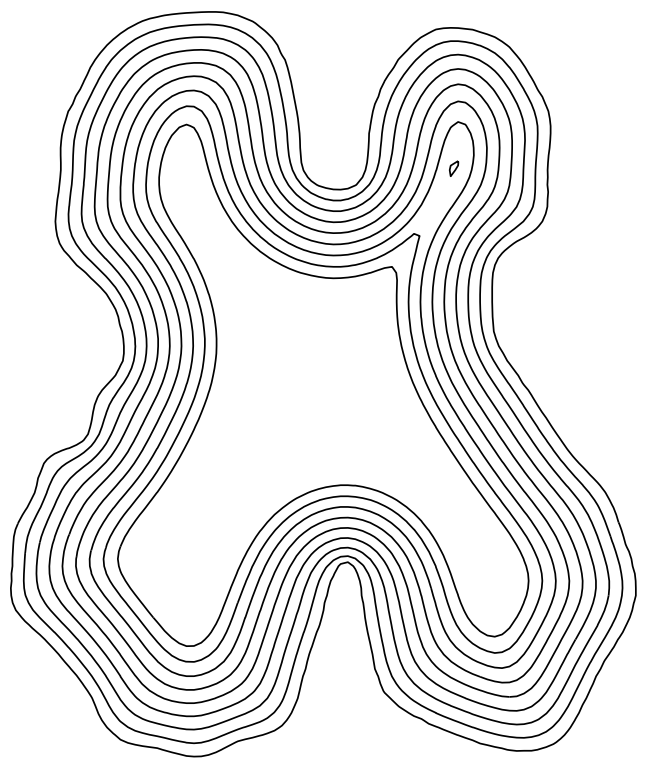

Figure 10. Level set method for $F=-1-2 \kappa$.

better than the Lagrangian method because it is stable but it is still not as good as the level set method due to its lack of smoothness (figure 9).

This method still uses a local representation of the front so when corners appear swallowtails develop in time, exactly as before. The difference consists in the fact that the scheme considered here is not unstable, or, at least, the oscillations which might appear due to the accumulation of small errors develop at later times than in the Lagrangian method for the same $\epsilon$ (see figures $9 \mathrm{a}$ and $\mathrm{b}$ ).

As it is shown in [18], the formation of sharp corners and the breaking of the curve are handled naturally by the level set method. Also, this method is stable (see figure 10). Comparing figures 8,9 and 10 we can see that, while the constant speed -1 acts as an advection term, contracting the curves, the term $\epsilon \kappa$ is a diffusive term which smooths out the high curvature regions and hence has a regularizing effect on the evolving curves. Also, we notice the similarities between the shape of the ventricular wall from figure $1 \mathrm{~b}$ and the curve from figure 11 which is just one of the evolved curves of figure $8 \mathrm{~b}$.

Thus we can conclude first that the level set method has better chances at successfully representing the evolution of the ventricular wall after shunt implantation. And secondly, based on our simulation using the level set method (figures 8b, 9c and 10) we can say that a catheter placed in the occipital horn (the lower side of the ventricular wall) has better chances of survival than in the frontal horn (the upper side of the ventricular wall), which is in agreement with the experimental study of [12].

\section{Discussion and conclusions}

A well recognized risk factor for ventricular CSF shunts failure in the treatment of hydrocephalus is obstruction

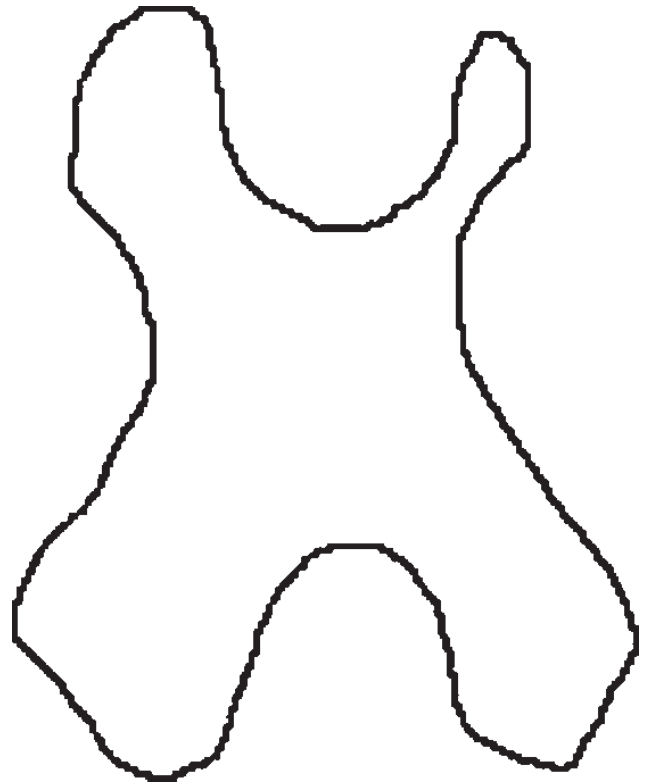

Figure 11. One of the evolving curves from figure $8 b$.

of the ventricular catheter and improper positioning of the catheter tip may play a significant role. Unfortunately, no scientifically sound method has been found so far to help the neurosurgeon find the optimal location; in fact, catheter location has been the source of a long-standing controversy in the treatment of hydrocephalus [9].

This paper reports some preliminary results on the analytic approach to this problem, which appears to be extremely promising. Using chain encoding, elliptical Fourier descriptors, and level set methods we are able to predict the ultimate shape of the ventricles in shunted hydrocephalus. Obviously, information of this type would greatly assist the neurosurgeon in choosing the optimal position of the ventricular catheter tip.

We have characterized our current results as preliminary for two main reasons. One of them is the restriction to the two-dimensional geometry of a single horizontal brain scan when, in reality, the problem is three-dimensional and the motion of the ventricular wall must be predicted. Another is the limitation implicit in our current assumption that each point of the ventricular wall moves at constant speed, when it is very likely that there is an effect of the local curvature on the ventricular motion. We are confident, however, that these problems do not pose insurmountable difficulties. For example, the extension to the three-dimensional case is straightforward and was carried out and recently reported by [23]. Regarding the problem of finding the ventricular decompression speed, one needs to construct a mathematical model which describes as well as possible the fluid/tissue interaction in the brain, i.e. the brain dynamics. This is a much more demanding problem and the reader interested in this topic is directed to [19] and the references within. 


\section{Acknowledgements}

This work was supported in part by a grant from the Hospital for Sick Children Foundation and by an NSERC Collaborative Health Research Grant. Also, we would like to thank the referees for their helpful suggestions.

\section{References}

[1] Netter, F., 1972, The CIBA Collection of Medical Illustrations. Nervos System, Vol. 1, CIBA.

[2] Milhorat, T., 1972, Hydrocephalus and Cerebrospinal Fluid (The Williams and Wilkins Comp.).

[3] Milhorat, T., 1978, Pediatric Neurosurgery, Contemporary neurology series: 16

[4] Czosnyka, M., Czosnyka, Z., Whitfield, P., Donovan, T. and Pickard, J., 2001, Age dependence of cerebrospinal pressurevolume compensation in patients with hydrocephalus, J. Neurosurg. 94, 482-486.

[5] Nolte, J., 1993, The Human Brain: An Introduction to Its Functional Anatomy (Mosby-Year Book Inc).

[6] Gjerris, F. and Borgesen, S., 1992, Current concepts of measurement of cerebrospinal fluid absorption and biomechanics of hydrocephalus, Adv. Tech. Stand. Neurosurg., 19, I45-177.

[7] Brandt, M., Bohan, T., Kramer, L. and Fletcher, J., 1994, Estimation of CSF, white and gray matter volumes in hydrocephalic children using fuzzy clustering of MR images, Comput. Med. Imaging Graph., 18(1), 25-34.

[8] Sood, S., Ham, S. and Canady, A., 2001, Current treatment of hydrocephalus, Neurosurg. Quart., 11, 36-44.

[9] Drake, J. and Sainte-Rose, C., 1995, The Shunt Book (Blackwell Science Inc).

[10] Aschoff, A., Kremer, P., Hashemi, B. and Kunze, S., 1999, The scientific history of hydrocephalus and its treatment, Neurosurg. Rev., 22, 67-93.
[11] Tuli, S., O’Hayon, G., Drake, J. and Kestle, J., 1998, Change of ventricular size and effect ventricular catheter placement of shunted hydrocephalus, Can. J. Neurol. Sci., 25:S43(Abstract).

[12] Tuli, S., O’Hayon, B., Drake, J., Clarke, M. and Kestle, J., 1999, Change in ventricular size and effect of ventricular catheter placement in pediatric patients with shunted hydrocephalus, Neurosurgery, 45(6), 1329-1335.

[13] Drake, J., Kestle, J., Milner, R., Cinalli, G., Boop, F., Piatt, J., Haines, S., Cochrane, D., Steinbok, P. and MacNeil, N., 1998, Randomized clinical trial of cerebrospinal fluid shunt valve design in pediatric hydrocephalus, Neurosurgery, 43, 294-305.

[14] Sainte-Rose, C., Hoffman, H. and Hirsch, J., 1999, Shunt: failure, Concepts Pediatr. Neurosurg., 9, 7-20.

[15] Read, D., 1990, From multivariate to qualitative measurement: representation of shape, Hum. Evol., 5, 417-429.

[16] Kuhl, F. and Giardina, C., 1982, Elliptic fourier features of a closed contour, Comput. Graph. Image Process., 18, 236-258.

[17] Dougherty, E. and Giardina, C., 1987, Image ProcessingContinuous to Discrete, Volume 1: Geometric, Transform and Statistical Methods (Prentice-Hall, Inc).

[18] Sethian, J., 1999, Level Set Methods and Fast Marching Methods (Cambridge University Press).

[19] Drapaca, C., Brain Biomechanics: Dynamical Morphology and Non-Linear Viscoelastic Models of Hydrocephalus, PhD Thesis, University of Waterloo.

[20] Smith, G.D., 1985, Numerical Solution of Partial Differential Equations: Finite Difference Methods, Oxford Applied Mathematics and Computing Science Series, Oxford University Press.

[21] Sethian, J., 1990, Numerical algorithms for propagating interfaces: Hamilton-Jacobi equations and conservation laws, J. Differ. Geom., 31, $131-161$.

[22] Osher, S. and Fedkiw, R., 2003, Level Set Methods and Dynamic Implicit Surfaces, Applied Mathematical Sciences (NewYork, Inc: Springer-Verlag), Vo1. 153.

[23] West, J., Application of the Level Set Method to Hydrocephalus: Simulating the Motion of the Ventricles, PhD Thesis, University of Waterloo. 


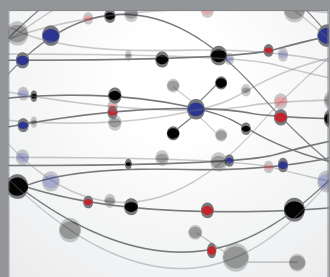

The Scientific World Journal
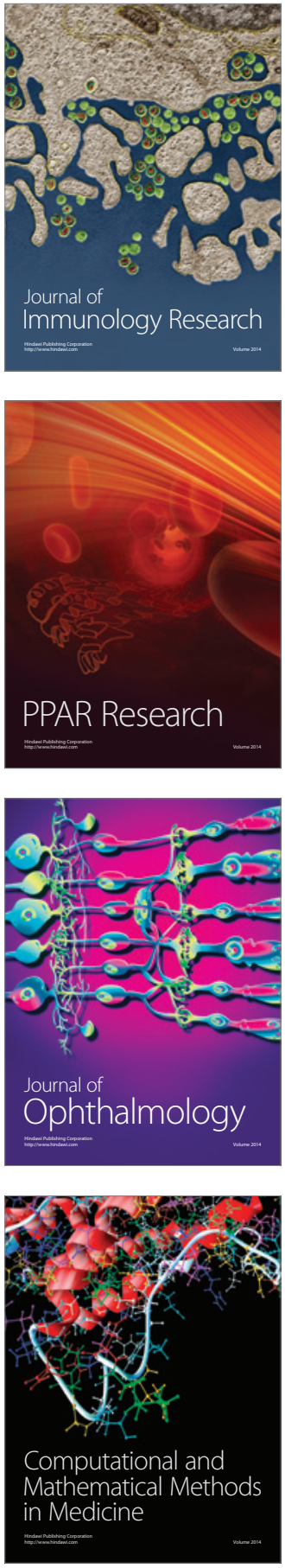

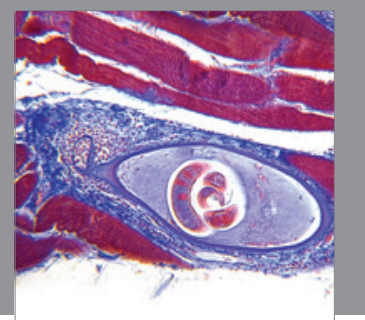

Gastroenterology

Research and Practice
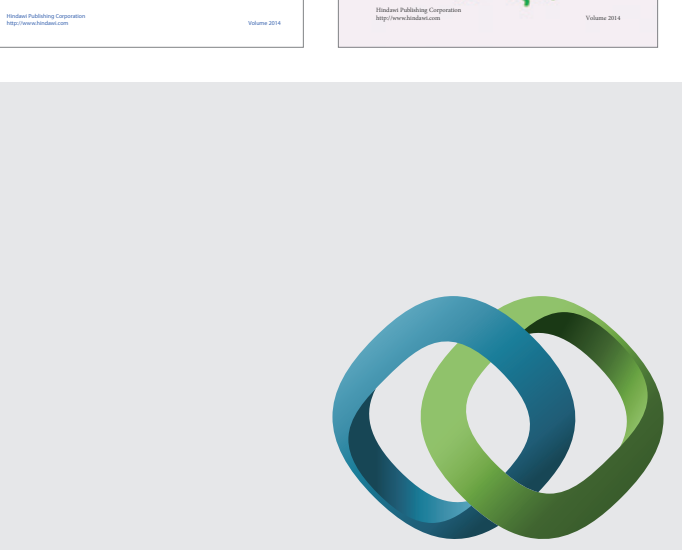

\section{Hindawi}

Submit your manuscripts at

http://www.hindawi.com
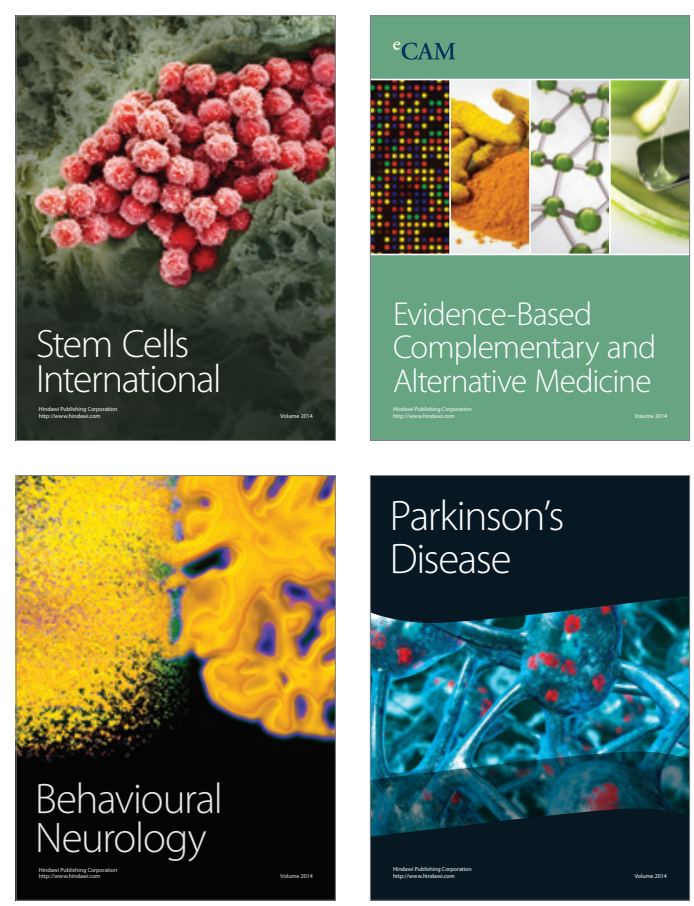

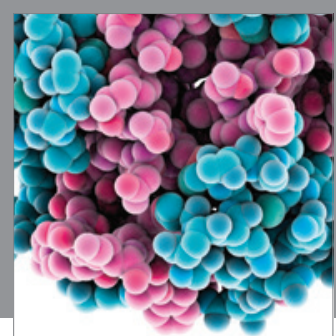

Journal of
Diabetes Research

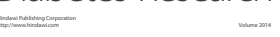

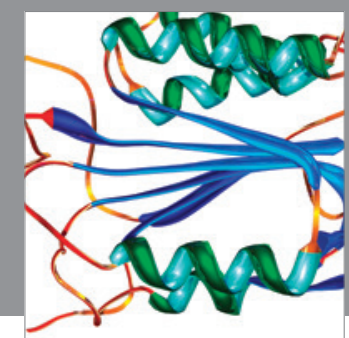

Disease Markers
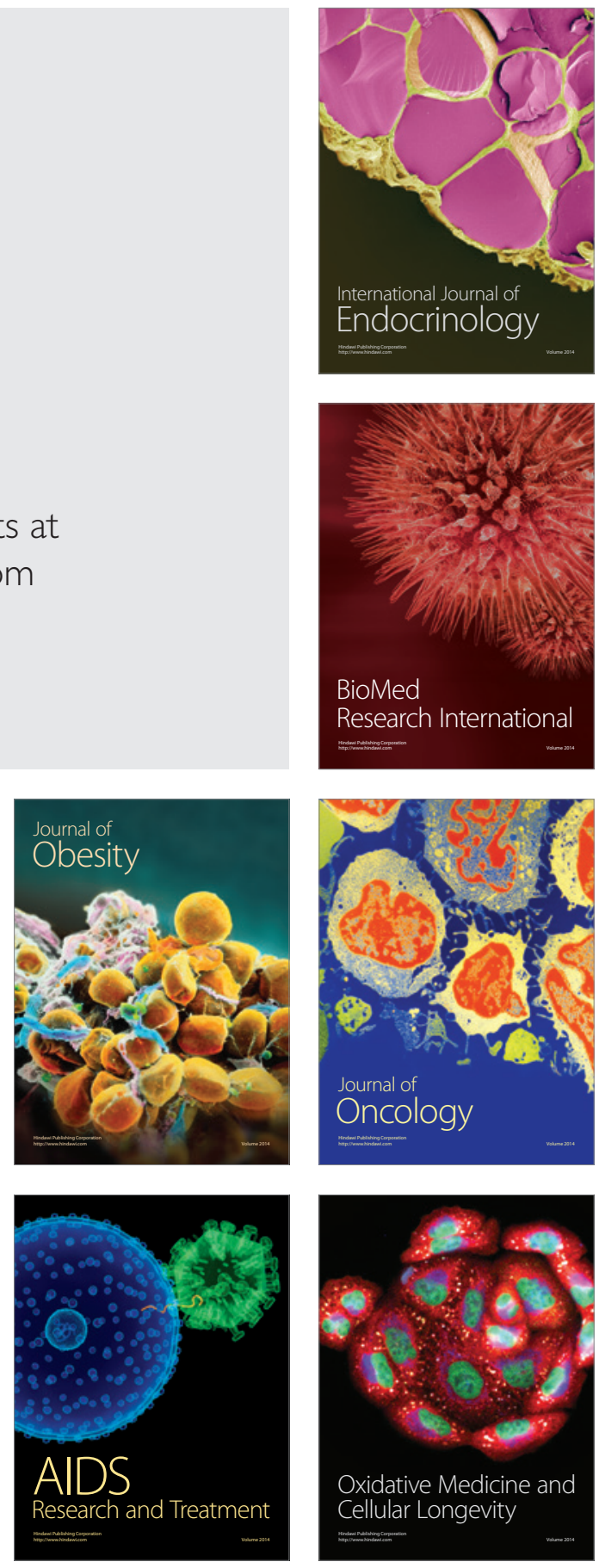